\title{
Multi-objective Day-ahead Scheduling of Microgrids Using Modified Grey Wolf Optimizer Algorithm
}

\author{
Mahshid Javidsharifia ${ }^{\mathrm{a}, 1}$, Taher Niknam ${ }^{\mathrm{a}, 2^{*}}$, Jamshid Aghaei ${ }^{\mathrm{a}, 3}$, Geev Mokryani ${ }^{\mathrm{b}, 4}$, Panagiotis Papado- \\ poulos, ${ }^{\mathrm{c}, 5}$ \\ ${ }^{a}$ Electrical and Electronics Engineering Department, Shiraz University of Technology, Shiraz, Iran \\ ${ }^{\mathrm{b}}$ School of Electrical Engineering and Computer Science, University of Bradford, Bradford BD7 1DP, UK \\ ${ }^{c}$ HIT Innovations, 2408 Egkomi, Nicosia, Cyprus \\ ${ }^{1}$ m.javidsharifi@sutech.ac.ir; ${ }^{2}$ niknam@sutech.ac.ir; ${ }^{3}$ aghaei@sutech.ac.ir; ${ }^{4}$ g.mokryani@bradford.ac.uk, \\ 5 p.papadopoulos@hit-innovations.com
}

\begin{abstract}
Investigation of the environmental/economic optimal operation management of a microgrid (MG) as a case study for applying a novel modified multi-objective grey wolf optimizer (MMOGWO) algorithm is presented in this paper. MGs can be considered as a fundamental solution in order for distributed generators' (DGs) management in future smart grids. In the multiobjective problems, since the objective functions are conflict, the best compromised solution should be extracted through an efficient approach. Accordingly, a proper method is applied for exploring the best compromised solution. Additionally, a novel distance-based method is proposed to control the size of the repository within an aimed limit which leads to a fast and precise convergence along with a well-distributed Pareto optimal front. The proposed method is implemented in a typical gridconnected MG with non-dispatchable units including renewable energy sources (RESs), along with a hybrid power source (micro-turbine, fuel-cell and battery) as dispatchable units, to accumulate excess energy or to equalize power mismatch, by optimal scheduling of DGs and the power exchange between the utility grid and storage system. The efficiency of the suggested algorithm in satisfying the load and optimizing the objective functions is validated through comparison with different methods, including PSO and the original GWO.
\end{abstract}

Keywords: Multi objective optimal operation management, Pareto optimal solution, Modified grey wolf optimizer, Micro-grid, Renewable energy sources

\section{Introduction}

Recent increases in energy prices and environmental concerns have led to the penetration of renewable distribution generators (DGs) in distribution systems [1]. Microgrid (MG) is a concept resulted from the need of reliable power systems with clean energy sources which can make it easy to have a satisfactory communication and optimal energy management of the power system [2]. A review of modelling, planning and energy management of an MG is presented in [3]. Since control and operation management of
MGs are midway through improvement, diverse techniques are proposed in order for optimization of these networks. Consequently, more precise energy source scheduling in MGs considering different objectives seems to be required. Various researches have been conducted dealing with optimal operation scheduling considering different constraints and objectives [4-12].

The choice of optimization technique depends on several different factors; therefore, different methods such as mathematical programming based optimization approaches and meta-heuristic algorithms can be

${ }^{*}$ Corresponding author. Tel: +98 713726 4121; Fax: +98 713735 5350; Email: niknam@ sutech.ac.ir 
proposed to solve problems $[13,14]$. The lambda iterative method, gradient projection method, linear/non-linear programming interior point methods, dynamic programming, etc. can be named as examples of mathematical programming based methods. On the other hand, meta-heuristic algorithms are proper alternatives to mathematical programming. In the case of solving multi-objective optimization problems, the major motivation of employing metaheuristic algorithms instead of mathematical programming approaches is the ability of meta-heuristics in finding different solutions in the Pareto optimal front in just one execution of the algorithm, while the mathematical programming methods apply a sequence of independent executions. Furthermore, meta-heuristic algorithms are not sensitive to the continuity and formation of the Pareto front [13].

In the MGs' environmentalleconomic management a certain number of DG units are supposed to supply the load while minimum levels of cost and emission are satisfied under considered constraints [12]. When minimization of both environmental pollutants' emission and energy cost comprise the objectives, the problem will be multi-objective, since these objective functions are conflicting. Multi-objective optimization approaches are developed for the sake of achieving Pareto optimal solutions of the conflicting objectives such that the operator is capable of making a trade-off among the set of optimal solutions. Some papers are devoted to the multi-objective economiclemission optimal operation of MGs, applying different meta-heuristic algorithms [5], [15-19]. A krill herd $(\mathrm{KH})$ algorithm is suggested in [5] for stochastic optimal operation management of a grid-connected MG. In [15] optimization of the environmental economic problem in $\mathrm{MG}$ is considered and multiobjective mesh adaptive direct search is presented to minimize the total cost. A multi-objective bi-level optimal operation model for distribution network and MGs is suggested in [16] and the problem is solved using a self-adaptive genetic algorithm and nonlinear programming. In [17] a $\theta$-PSO algorithm is applied to deal with the MG's energy management problem. Authors in [18] proposed a $\theta$-krill herd algorithm. A multi-objective PSO is used in [19] in order to optimize MG's short-term performance. The superiority of an optimization algorithm in solving multiobjective problems is revealed from its robustness and fast convergence to a well-distributed Paretooptimal front over the course of time. In comparison with other meta-heuristic algorithms, the grey wolf optimizer (GWO) algorithm, introduced by Mirjalili et al, manifests approximately proper search speed and convergence in solving some optimization problems [20]. In this paper, in order to investigate the multi-objective optimal operation management (MOOM) problem, a modified multi-objective GWO (MMOGWO) algorithm is proposed. Most of algorithms are needy to onerous tuning process of control parameters which is not required in MMOGWO that makes it superior among all other algorithms. Accordingly, the following objectives are met in the paper:

(i) A novel algorithm is proposed comprising three modifications. The first modification is in the size of population which leads to a variable population. Consequently, trapping in local optima is avoided and the algorithm's convergence speed is increased. Two other modifications are imposed in the mutations as is described in Section 4.3, which lead to the increase of the accuracy and convergence capability of the algorithm.

(ii) A novel method for controlling the size of repository is applied such that the algorithm's speed improved while the search space becomes immense which leads to finding the optimum global (best compromised) solution faster and more precisely.

(iii) Since the ON/OFF states of DGs are taken in to account, a mixed-integer problem is solved in the article. Two different objective functions are assumed. An exquisite Pareto front of optimal solutions is achieved while the computational time is very low. The effectiveness of the proposed approach is affirmed by applying it on the typical MG of [12]. Additionally, the robustness of the algorithm, as its other outstanding feature, is highlighted through the simulation results.

\section{Problem formulation}

In the considered MOOM problem, optimal allocation of power generation set points besides proper On\Off states of DG units are defined such that the objective functions, namely the operating cost and emission of the MG, are minimized while several constraints are satisfied [12].

\subsection{Cost and emission minimization}

In order to consider total operation cost as the first objective function the following should be satisfied: 


$$
\begin{aligned}
& F_{1}(X)=\sum_{t=1}^{T} \operatorname{Cost}^{t}=\sum_{t=1}^{T}\left\{\sum _ { i = 1 } ^ { N _ { D G } } \left[u_{i}^{t} \cdot\left(P_{D G i}^{t}\right) \cdot B_{D G i}^{t}+\right.\right. \\
& \left.S U C_{D G i} \cdot u_{i}^{t} \cdot\left(1-u_{i}^{t-1}\right)+S D C_{D G i} \cdot u_{i}^{t-1} \cdot\left(1-u_{i}^{t}\right)\right] \\
& \left.+\sum_{r=1}^{N_{\text {RES }}}\left[P_{R E S_{r}}^{t} \cdot B_{R E S_{r}}^{t}\right]+\sum_{s=1}^{N_{\text {Batt }}}\left[P_{\text {Batt }}^{t} \cdot B_{\text {Batt }}^{t}\right]+P_{\text {Grid }}^{t} \cdot B_{\text {Grid }}^{t}\right\} \\
& \stackrel{\mu}{X}=\left[\stackrel{\mu}{U}_{D G}, \stackrel{P_{D G}}{P_{D}}, \stackrel{\mu}{P}_{\text {Batt }}, \stackrel{\mu}{P}_{\text {Grid }}\right]_{1 \times n} \\
& n=\left[\left(\left(2 \times N_{D G}\right)+N_{\text {Batt }}\right)+1\right] \times T
\end{aligned}
$$

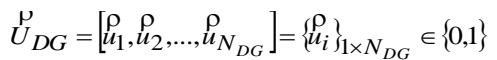

$$
\begin{aligned}
& \rho_{i}=\left\{u_{i}^{1}, u_{i}^{2}, \ldots, u_{i}^{t}, \ldots, u_{i}^{T}\right\} \quad i=1,2, \ldots, N_{D G} \\
& \stackrel{\rho}{P}_{D G}=\left[\stackrel{\rho}{P}_{D G_{1}}, \stackrel{\rho}{P}_{D G_{2}}, \ldots, \stackrel{\rho}{P}_{D G_{N_{D G}}}\right] \\
& \left.\stackrel{\rho}{P}_{D G i}=\left[P_{D G i}^{1}, P_{D G i}^{2}, \ldots, P_{D G i}^{t}, \ldots, P D_{G i}^{T}\right\rfloor\right] \quad i=1,2, \ldots, N_{D G} \\
& \stackrel{\rho}{P_{\text {Batt }}}=\left[\begin{array}{ll}
\rho_{\text {Batt }_{1}}, \stackrel{\rho}{P}_{\text {Batt }_{2}}, \ldots, \stackrel{\rho}{P}_{\text {Batt }_{N_{\text {Bat }}}}
\end{array}\right] \\
& \stackrel{\rho}{P}_{\text {Batt }, s}=\left\lfloor P_{\text {Batt }, s}^{1}, P_{\text {Batt }, s}^{2}, \ldots, P_{\text {Batt }, s}^{t}, \ldots, P_{\text {Batt }, s}^{T}\right\rfloor s=1,2, \ldots, N_{\text {Batt }} \\
& \stackrel{\rho}{P}_{R E S}=\left[\rho_{P_{R E S}}, \rho_{R E S_{2}}, \ldots, \rho_{R E S_{N_{R E S}}}\right] \\
& \stackrel{\rho}{P}_{R E S_{r}}=\left[P_{R E S_{r}}^{1}, P_{R E S_{r}}^{2}, \ldots, P_{R E S_{r}}^{t}, \ldots, P_{R E S_{r}}^{T}\right] ; r=1,2, \ldots, N_{R E S} \\
& \stackrel{\rho}{P}_{\text {Grid }}=\left[P_{\text {Grid }}^{1}, P_{\text {Grid }}^{2}, \ldots, P_{\text {Grid }}^{t}, \ldots, P_{\text {Grid }}^{T}\right]
\end{aligned}
$$

in which $X$ is the vector of design variables, and $n$ is the number of design variables. $T$ is the total number of hours. The total number of dispatchable generations and storage units (battery) along with the number of RESs are $N_{D G}, N_{B a t t}$ and $N_{R E S}$, respectively. $u_{i}^{t}$ is applied to imply the ON/OFF states of $i^{\text {th }}$ dispatchable DG during each hour of the day. $P_{D G i}^{t}$, $P_{R E S_{r}}^{t}$ and $P_{B a t t, s}^{t}$ represent the real output powers $(\mathrm{kWh})$ of the $i^{\text {th }} \mathrm{DG}, r^{\text {th }} \mathrm{RES}$ and $s^{\text {th }}$ storage at time $t$, respectively. The active power which is bought (sold) from (to) the utility at time $t$ is demonstrated by $P_{\text {Grid }}^{t} \cdot B_{D G i}^{t}, B_{R E S_{r}}^{t}, B_{B a t t, s}^{t}$ and $B_{\text {Grid }}^{t}$ are respectively bids of dispatchable DGs, RESs, storage devices and the utility grid at hour $t(€ / \mathrm{kWh}) . S U C_{D G i}$ and $S D C_{D G i}$ are the start-up and shut-down cost for $i^{\text {th }}$ dispatchable DG. $\quad\left(P_{D G i}^{t} \cdot B_{D G i}^{t}\right) \quad, \quad\left(P_{\text {Batt }_{s}}^{t} \cdot B_{\text {Batt }_{s}}^{t}\right)$ and $\left(P_{\text {Grid }}^{t} \cdot B_{\text {Grid }}^{t}\right)$ represent operational cost of dispatchable DGs, battery and cost of power exchange between the $\mathrm{MG}$ and utility $(€)$, respectively. It should be mentioned that $D G$ demonstrates the dispatchable units including fuel cell (FC) and microturbine (MT), while Grid and Batt are abbreviated forms of the utility grid and the battery, respectively. The WT and PV are shown with RES.

Note that the utility has to buy all electrical power produced by RES units, consequently RESs' output powers $\left(P_{R E S, r}^{t}\right)$ are not included in the design variables' vector.

As the second objective, the environmental pollutants should be minimized as the following [12]:

$$
\begin{aligned}
& F_{2}(\stackrel{\rho}{\rho})=\sum_{t=1}^{T} \text { Emission }^{t}=\sum_{t=1}^{T}\left\{\sum_{i=1}^{N_{D G}}\left[u_{i}^{t} \cdot\left(P_{D G i}^{t}\right) \cdot E_{G i}^{t}\right]+\right. \\
& \left.\sum_{s=1}^{N_{\text {Batt }}}\left[P_{\text {Batt }}^{t} \cdot E_{\text {Batt }}^{t}\right]+P_{\text {Grid }}^{t} \cdot E_{\text {Grid }}^{t}\right\}
\end{aligned}
$$

where $E_{D G i}^{t}, E_{\text {Batt }}^{t}$ and $E_{\text {Grid }}^{t}$ are the amount of pollutants emission $(\mathrm{kg} / \mathrm{kWh})$ for each generator, storage device and utility at hour $t$, respectively. These variables are described as follows [12-19]:

$$
\begin{aligned}
& E_{D G i}^{t}=C O_{2_{D G_{i}}^{t}}^{t}+S O_{2_{D G_{i}}^{t}}^{t}+N O_{x_{D G_{i}}}^{t} \\
& E_{\text {Batt }}^{t}=C O_{2_{\text {Batt,s }}}^{t}+S O_{2_{\text {Batt,s }}}^{t}+N O_{x_{\text {Batt,s }}}^{t} \\
& E_{\text {Grid }}^{t}=C O_{2_{\text {Grid }}^{t}}^{t}+S O_{2_{\text {Grid }}}^{t}+N O_{x_{\text {Grid }}}^{t}
\end{aligned}
$$

where $C O_{2_{D G_{i}}}^{t}, S O_{2_{D G_{i}}}^{t}$ and $N O_{x_{D G_{i}}}^{t}$ are the amounts of $\mathrm{CO}_{2}, \mathrm{SO}_{2}$ and $\mathrm{NO}_{x}$ emission from $i^{\text {th }}$ DG source at hour $t ; \mathrm{CO}_{2_{\text {Batt,s }}}^{t}, S \mathrm{~S}_{2_{\text {Batt,s }}^{t}}^{t}$ and $N O_{x_{\text {Batt,s }}}^{t}$ are the amounts of $\mathrm{CO}_{2}, \mathrm{SO}_{2}$ and $\mathrm{NO}_{x}$ emission from the $s^{\text {th }}$ storage unit at hour $t$ of the day, and $\mathrm{CO}_{2_{\text {Grid }}}^{t}, S O_{2_{\text {Grid }}}^{t}$ and $\mathrm{NO}_{x_{\text {Grid }}}^{t}$ are the amounts of $\mathrm{CO}_{2}, \mathrm{SO}_{2}$ and $\mathrm{NO}_{x}$ emission from the utility at hour $t$, respectively.

\subsection{Constraints}

\section{- Power balance}

One of the most important requirements in $\mathrm{MG}$ management is the balance of electricity demand and supply, hence [12]:

$\sum_{i=1}^{N_{D G}}\left[u_{i}^{t} \cdot P_{D G i}^{t}\right]+\sum_{r=1}^{N_{\text {RES }}}\left[P_{R E S_{r}}^{t}\right]+\sum_{s=1}^{N_{\text {Batt }}}\left[P_{\text {Batt }}^{t}\right]+P_{\text {Grid }}^{t}=P_{L D}^{t}$

where $P_{L D}^{t}$ is the total MG load at hour $t$.

-Battery limits 
In order to consider the limitation on charge and discharge rates of the storage devices during each time intervals, along with limits on the state of charge (SOC) of each storage device the following equation and constraints are mentioned for a typical battery [21]:

$W_{\text {Batt }_{s, \min }} \leq W_{\text {Batt }_{S}}^{t} \leq W_{\text {Batt }_{s, \text { max }}}$ 
where $W_{\text {Batts }}^{t}$ is the SOC of the $s^{\text {th }}$ storage at the end of one-hour interval which is associated to the time $t$ as the following:

$$
\begin{aligned}
& W_{\text {Batt }}^{t}=W_{\text {Batt }}^{t-1}+\eta_{c} P_{c h} \cdot \Delta t-\frac{1}{\eta_{d}} P_{\text {disch }} \cdot \Delta t \\
& P_{c h}^{t} \leq P_{\text {ch, } \max } \\
& P_{\text {disch }}^{t} \leq P_{\text {disch, } \text { max }}
\end{aligned}
$$

in which $W_{\text {Batt }}^{t}$ and $W_{\text {Batt }}^{t-1}$ are the amounts of energy stored inside the battery at hours $t$ and $t$-1, respectively. During a definite period of time $(\Delta t), P_{c h}\left(P_{\text {disch }}\right)$ is the permitted rate of charge (discharge), while $\eta_{c}\left(\eta_{d}\right)$ is the efficiency of the battery during charge (discharge) process. The lower and upper limits of amounts of energy storage inside the battery are

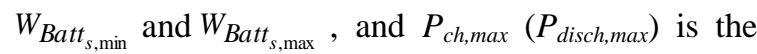
maximum rate of battery charge (discharge) during each time interval $\Delta t$.

\section{- $\quad$ Real power constraint}

Power generations for each dispatchable DG are limited as:

$$
u_{i}^{t} \cdot P_{D G_{i, \min }}^{t} \leq P_{D G_{i}}^{t} \leq u_{i}^{t} \cdot P_{D G_{i, \max }}^{t}
$$

The power exchange with utility grid is constrained as follows:

$$
P_{\text {Grid,min }}^{t} \leq P_{\text {Grid }}^{t} \leq P_{\text {Grid, } \text { max }}^{t}
$$

Constraints on the rate of charge and discharge of the battery during an hour are considered as the following:

$$
P_{\text {Batt }_{s}, \min }^{t} \leq P_{\text {Batt }_{s}}^{t} \leq P_{\text {Batt }_{s}, \max }^{t}
$$

\section{Fundamentals of multi-objective optimization}

\subsection{Characterization of multi-objective optimization}

In a typical multi-objective problem, a number of objective functions are simultaneously optimized. In most of the situations, these objective functions are conflict which is a barrier to select an optimum solution for all the objective functions. In most cases, multi-objective problems have more than one optimal solution, which are called the non-dominated solutions. Within the whole search space, the nondominated solutions are expressed as Pareto-optimal which establish the Pareto-optimal set or Paretooptimal front. Considering a multi-objective minimi- zation problem, while meeting a number of equality and inequality constraints, a solution $X_{1}$ dominates $X_{2}$ if the following conditions are satisfied [13, 14]:

$$
\begin{aligned}
& \forall j \in\{1,2, \ldots, n\}, f_{j}\left(X_{1}\right) \leq f_{j}\left(X_{2}\right) \\
& \exists k \in\{1,2, \ldots, n\}, f_{k}\left(X_{1}\right)<f_{k}\left(X_{2}\right)
\end{aligned}
$$

where $f_{i}(X)$ is the $i^{\text {th }}$ objective function, $X$ is the vector of the optimization variables, and $n$ is the number of objective functions $[13,14]$. In order to assess the suitable particles to be stored in the repository of non-dominated solutions, the concept of Paretooptimal is employed [13, 14].

\subsection{Controlling the size of repository}

Since the size of repository is limited, a finite number of solutions can be accumulated. In this paper, repository size is controlled through a novel distance-based method.

When the number of non-dominated solutions in the repository exceeds a predefined value, namely $N_{L}$, they are sorted ascending according to one of the objective functions, and the first and the last of the sorted non-dominated solutions are assumed as $A$ and $B$, respectively. The pseudo-code of the proposed method for controlling the size of repository is as shown in Table 1 . In this table $N_{\text {non-dom }}$ is the number of non-dominated solutions, $f_{i}$ is the objective function and $M$ is the number of objective functions.

$$
\text { Table } 1
$$

The pseudo-code of the proposed method for controlling the size

$$
\begin{aligned}
& \text { epsilon }=\frac{\sqrt{\sum_{m=1}^{M}\left(f_{A_{m}}-f_{B_{m}}\right)^{2}}}{N_{L}} \\
& \text { For } i=2 \text { to } N_{n o n-d o m} \\
& \text { distance }=\sqrt{\sum_{m=1}^{M}\left(f_{1_{m}}-f_{i_{m}}\right)^{2}} \\
& \text { End } \\
& 1=1 \\
& \text { For } i=1 \quad \text { to } N_{L} \\
& K=\text { find }\left((i-1)^{*}\right. \text { epsilon<distance and distance <i*epsilon) } \\
& \text { If length } K \sim=0 \\
& Y=K \text { (end) } \\
& 1=1+1 \\
& \text { End } \\
& \text { End }
\end{aligned}
$$




\subsection{Selecting the best compromised solution}

As was mentioned, the Pareto front should be scattered uniformly. Additionally, considering each objective function to be minimized independently, the best solution of each should be obtained. In order to achieve these goals, particles should be refrained to accumulate in populated domains. Hence, the proposed method of [21] is applied to improve the properties of Pareto optimal front and to select the global best compromised solution. In this technique, objective functions are normalized such that relatively equal significance is provided to the both objectives as follows:

$$
\operatorname{Min} \phi^{M}=\omega_{1}\left[\frac{\sum_{i=1}^{N_{\text {non-dom }}} f_{1_{i}} f_{1}^{\min }}{f_{1}^{\max }-f_{1}^{\min }}\right]+\omega_{2}\left[\frac{\sum_{i=1}^{N_{\text {non-dom }}} f_{2_{i}-f_{2}^{\min }}}{f_{2}^{\max }-f_{2}^{\min }}\right]
$$

where $f_{1}$ and $f_{2}$ are objective functions cost and emission, respectively. Initial guess for $\omega_{1}$ and $\omega_{2}$ is equal to 0.5 .

\section{Modified GWO algorithm}

\subsection{A brief overview on original GWO algorithm}

A swarm-intelligence algorithm impressed by the hierarchical hunting manner of grey wolves, namely GWO, is presented by Mirjalili et al. [20]. The social hierarchy of grey wolves is classified in four groups including the group leader, alpha $(\alpha)$, as the first level, beta $(\beta)$ as the second level who cooperates alpha in decision making, while the third best solution is called delta $(\delta)$ which comes after alpha and beta but leads the fourth hierarchy, omega $(\omega)$, which should defer to other three dominant levels. The hunting mechanism of grey wolves is as follows [20]:

(i) Tracking, chasing, and approaching the prey.

(ii) Pursuing, encircling, and harassing the prey until it stops moving.

(iii) Attacking the prey

In the optimization process $\omega \mathrm{s}$ randomly update their positions around the prey according to the estimated position of the prey by $\alpha, \beta$ and $\delta$. The encircling is then performed as follows:

$$
\begin{aligned}
& D=\left|C . X_{p, k}-X_{k}\right| \\
& X_{k+1}=X_{p, k}-A . D
\end{aligned}
$$

$k$ indicates the current iteration, $X_{p, k}$ is the position vector of the prey, $X_{k}$ is the position vector of a grey wolf, $D$ is the distance between $X_{p, k}$ and $X_{k}$, while $A$ and $C$ represent the discrimination weight coefficient of search agent, and random mutation coefficients, respectively and are calculated as following:

$$
A=\left(2 r_{1}-1\right) a
$$

$C=2 r_{2}$

where $r_{1}$ and $r_{2}$ are random vectors in [0,1], $a$ is linearly decreased from 2 to 0 over the course of iteration.

Hunting the prey as the last step of the procedure is guided by $\alpha$, while $\beta$ and $\delta$ participate in this step. $\alpha, \beta$ and $\delta$ have better knowledge about the potential position of the prey. The position of each grey wolf is updated as follows:

$$
\begin{aligned}
& D_{\alpha}=\left|C_{1} \cdot X_{\alpha, k}-X_{k}\right| \\
& D_{\beta}=\left|C_{2} \cdot X_{\beta, k}-X_{k}\right| \\
& D_{\delta}=\left|C_{3} \cdot X_{\delta, k}-X_{k}\right| \\
& X_{1}=X_{\alpha}-A_{1} \cdot\left(D_{\alpha}\right), X_{2}=X_{\beta}-A_{2} \cdot\left(D_{\beta}\right), X_{3}=X_{\delta}-A_{3} \cdot\left(D_{\delta}\right) \\
& X_{k+1}=\frac{1}{3}\left(X_{1}+X_{2}+X_{3}\right)
\end{aligned}
$$

where $A_{1}, A_{2}$ and $A_{3}$ are respectively decisive weight coefficients of $\alpha, \beta$ and $\delta$, values of which decrease progressively.

\subsection{Multi-objective GWO (MGWO)}

In this paper in order to investigate a bi-objective problem to optimize both the total cost and emission simultaneously, a multi-objective GWO (MOGWO) should be applied. Accordingly, $x_{\alpha}, x_{\beta}$ and $x_{\delta}$ are not fixed for each member and are selected randomly from the repository as is described. If the number of non-dominated solutions in the repository is more than three, after being sorted according to one of the objective functions the repository is divided into three equal sections. $X_{\alpha}$ is selected randomly from the first section which is from the first nondominated solution in the repository up to the ${\frac{N_{L}}{3}}^{t h}$, $X_{\beta}$ is selected from $\left(\frac{N_{L}}{3}+1\right)^{\text {th }}$ non-dominated solution up to ${\frac{2 N_{L}}{3}}^{\text {th }}$ non-dominated solution, and $X_{\delta}$ is chosen from the last section which is from the $\left(\frac{2 N_{L}}{3}+1\right)^{\text {th }}$ non-dominated solution to the last one. 
This procedure leads to an intelligent selection of $X_{\alpha}, X_{\beta}$ and $X_{\delta}$ where all three sections of the repository take part in the output Pareto front and consequently the actual Pareto optimal set is obtained.

\subsection{Modified multi-objective GWO algorthim (MMOGWO)}

In order to achieve a real Pareto optimal front in the MOOM problem, the original GWO should be enhanced. Consequently, three modifications, one in the size of population and the two others in the mutations, are augmented to improve the convergence ability and the accuracy of the approach.

\section{- Modification I}

To increase the convergence speed of the algorithm, the size of population is considered variable and changes as follows:

$$
N=\operatorname{round}\left(\frac{\left(N_{\max }-N_{\min }\right) \times \text { iteration }}{\text { iter }_{\max }}+N_{\min }\right)
$$

where $N_{\min }$ and $N_{\max }$ are the minimum and maximum populations respectively, and iter $_{\max }$ is the maximum number of iteration. A prominent feature of the proposed modification is that the selection of a variable population size leads to the increase of the population in each iteration, therefore, the population size is not fixed which helps to avoid trapping in local optima. As a result, the accuracy and convergence capability of the algorithm will improve.

\section{-Modification II}

In addition to the described modification in the previous section, the second modification is applied to improve the accuracy of the proposed approach. Five constants $k_{1} \neq k_{2} \neq k_{3} \neq k_{4} \neq k_{5}$, unequal to $i$, are chosen randomly from the population, while three constants $k_{1}^{\prime} \neq k_{2}^{\prime} \neq k_{3}^{\prime}$ are selected from the repository as follows: If the number of non-dominated solutions in the repository is more than three, after being sorted according to one of the objective functions the repository is divided into three sections. $k_{1}^{\prime}$ is selected randomly from the first section which is from the first non-dominated solution in the repository to the ${\frac{N_{L}}{3}}^{\text {th }}, k_{2}^{\prime}$ is selected from $\left(\frac{N_{L}}{3}+1\right)^{\text {th }}$ nondominated solution up to $\frac{2 N}{3}^{\text {th }}$ non-dominated solution, and $k_{3}^{\prime}$ is chosen from the last section which is from the $\left(\frac{2 N_{L}}{3}+1\right)^{\text {th }}$ non-dominated solution to the last one. Four mutations $\left({\stackrel{\mu}{X_{m u t_{l}}}}_{l}, l=1,2,3,4\right)$ are defined as:

$$
\begin{aligned}
& \stackrel{\text { as: }}{\stackrel{\mu}{X}_{m u t 1}}=\stackrel{\mu}{X}_{k_{1}}+\operatorname{rand1}(.) \times\left(\tilde{X}_{k_{2}}-\tilde{X}_{k_{3}}\right) \\
& \dot{X}_{\text {mut } 2}=\stackrel{\mu}{X}_{\text {mut } 1}+\operatorname{rand} 2(.) \times\left(\dot{X}_{\text {best }}-\stackrel{\mathcal{X}}{\text { worst }}\right)
\end{aligned}
$$

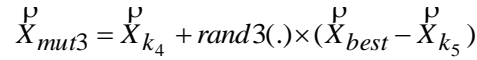

$$
\begin{aligned}
& \tilde{X}_{m u t 4}=\tilde{X}_{k_{1}^{\prime}}+\operatorname{rand} 4(.) \times\left(\tilde{X}_{k_{2}^{\prime}}-\tilde{X}_{k_{3}^{\prime}}\right)
\end{aligned}
$$

where after sorting the repository, the first nondominated solution is selected as $X_{\text {best }}$ and in each iteration the best non-dominated solutions are selected in turn as $X_{\text {best }}$ such that all repository members take part in the population generation, while $X_{\text {worst }}$ is the most dominated solution in the population.

\section{-Modification III}

In order to increase the convergence speed of the algorithm the third modification is augmented according to the pseudo code of Table 2.

\section{Application of the proposed method}

In order to apply the proposed algorithm on the MOOM problem in an MG, the following steps should be taken.

Step 1: Initialize population size, number of design variables and termination criterion. Problem information including MG properties, beside bids and power information of DGs, storages and utility, hourly wind-turbine (WT) and photovoltaic (PV) power forecasts, emission coefficients are specified. The initial charge of the battery is also defined in this step Step 2: Since a mixed integer problem is considered in this paper, two types of variables, binary and continuous, are assumed. For states of generators as binary variables, $U_{i}$ s according to Eq. (2) are generated as follows:

$$
u_{i}^{t}=\operatorname{round}\left(\operatorname{rand}(.) \times\left(u_{i, \max }^{t}-u_{i, \min }^{t}\right)+u_{i, \min }^{t}\right)
$$

However, in order to consider the states of all units, $U_{i}^{t}$ should satisfy the following condition for all hours:

$$
\begin{aligned}
& \sum_{i=1}^{N_{D G}}\left[u_{i}^{t} \cdot P_{D G_{i, \min }}^{t}\right]+\sum_{r=1}^{N_{R E S}}\left[P_{R E S_{r, \min }}^{t}\right]+\sum_{s=1}^{N_{\text {Batt }}}\left[P_{\text {Batt }}^{t}\right]+P_{s, \min }^{t} \leq P_{\text {Grid }} \text { min }_{\text {Load }} \\
& \leq \sum_{i=1}^{N_{D G}}\left[u_{i}^{t} \cdot P_{D G_{i, \max }}^{t}\right]+\sum_{r=1}^{N_{R E S}}\left[P_{R E S_{r, \max }}^{t}\right]+\sum_{s=1}^{N_{B a t t}}\left[P_{B a t t_{s, \max }}^{t}\right]+P_{G r i d_{\max }}^{t}
\end{aligned}
$$

If Eq. (23) is satisfied, a random population for continuous variables based on the achieved $U_{i}$ s and according to Eq. (2) must be generated as Eq. (24). 
Table 2

The pseudo-code of modification III.

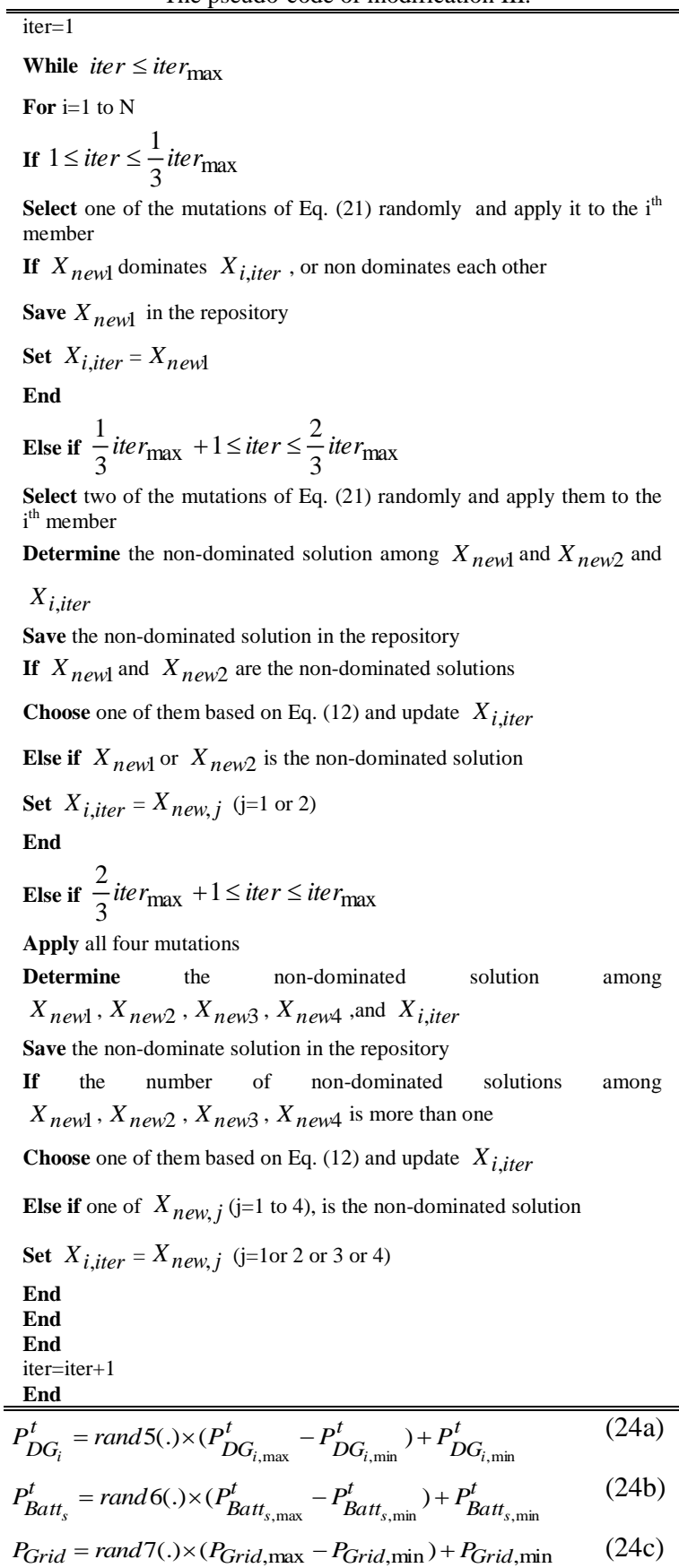

while the power constraints in Eqs. (8-10) should be satisfied.

Since in the considered problem some limitations, such as battery constraints, depend on previous and future hours, constraints change in different hours of the day as follows:

$$
\begin{aligned}
& W_{\text {disch,Batt }}^{t+1}=\max \left\{\left(\frac{W_{\text {Batt }}^{t}-\Delta t P_{\text {Batt }}^{t}}{\eta_{d}}\right), W_{\text {Batt }, \text { min }}\right\}, t=1, \ldots, T \\
& W_{c h, \text { Batt }}^{t+1}=\min \left\{\left(W_{\text {Batt }}^{t}-\Delta t P_{\text {Batt }}^{t}, \eta_{c}\right), W_{\text {Batt }, \text { max }}\right\}, t=1, \ldots, T \\
& P_{c h, \text { max }} \leq P_{\text {Batt }}^{t} \leq P_{\text {disch, } \max }, t=1, \ldots, T \\
& P_{\text {disch, } \max }^{t}=\min \left\{\left(P_{\text {Batt }, \text { max }}, \frac{\left(W_{\text {Batt }}^{t}-W_{\text {Batt,min }}\right) \eta_{d}}{\Delta t}\right)\right\}, t=1, \ldots, T \\
& P_{c h, \max }^{t}=\max \left\{\left(P_{\text {Batt,min }}, \frac{\left(W_{\text {Batt }}^{t}-W_{\text {Batt,max }}\right)}{\left(\eta_{c} \Delta t\right)}\right)\right\}, t=1, \ldots, T
\end{aligned}
$$

The limitation of the released energy in the discharging mode and the stored energy in charging mode are mentioned in Eqs. (25) and (26), respectively, while Eq. (28) demonstrates the power discharged by the battery in the discharge mode, and the power charged by the grid in the charging mode. Using Eqs. (28) and (29), the maximum and minimum rates of charging and discharging mode can be calculated [22].

Step 3: Check the power balance violation for each particle. In order to meet the equality constraint, following steps should be carried out:

Step 3.1: $\mathrm{t}=1$

Step 3.2: Calculate power balance violation:

$$
P_{V L T D}^{t}=\left(\sum_{i=1}^{N_{D G}}\left[u_{i}^{t} \cdot P_{D G i}^{t}\right]+\sum_{s=1}^{N_{\text {Batt }}}\left[P_{\text {Batt }}^{t}\right]+P_{\text {Grid }}^{t}\right)-\sum_{d=1}^{N_{d}} P_{L D}^{t}
$$

Step 3.3: If $P_{V L T D}^{t} \cong 0$ and $t \leq T$ then set $t=t+1$ and go to Step 3.2; otherwise If $P_{V L T D}^{t} \cong 0$ and $\mathrm{t}=\mathrm{T}$, go to Step 4 ; otherwise If $P_{V L T D}^{t} \neq 0$ select a generated unit randomly (battery, DGs or grid).

Step 3.4: Add $P_{V L T D}^{t}$ to the power of the selected generated unit.

Step 3.5: Check the result with upper and lower limits of the units' powers, if it violates the upper limit, fix it to the upper limit and if it violates the lower limit, fix it to the lower limit. Go to Step 3.2. Step 4: Calculate the objective functions for the initial population.

Step 5: Determine the non-dominated solutions in the population and store them in the repository.

Step 6: Choose ' $X_{\text {best }}$ ' and ' $X_{\text {worst }}$ ' as described in Section 4.3.

Step 7: Initialize the size of population based on Eq. (20).

Step 8: Select $i^{\text {th }}$ individual from population. Step 9: Apply GWO according to Eqs. (13-19). 
Step 10: Apply mutations as described in Section 4.3. Step 11: If all members are selected, go to Step 13, otherwise set $i=i+1$ and got to Step 9 .

Step 12: Update the number of population according to Eq. (20).

Step 13: Determine the non-dominated solutions in the new population.

Step 14: Update the repository based on new and old non-dominated solutions.

Step 15: If the number of non-dominated solutions is more than a predefined value, $N_{L}$, control the size of repository as mentioned in Section 3.2.

Step 16: Control the termination criterion, if it is satisfied, terminate the algorithm, otherwise, set iteration=iteration +1 and turn to Step 6.

Step 17: Report the best compromised solution which is achieved using the technique proposed in Section 3.3.

The flowchart of the proposed algorithm is illustrated in Figure 1.

\section{Simulation results}

The effectiveness of the proposed MMOGWO algorithm is verified in this section where two cases are investigated. The MG of Figure 2 is considered as the test system. A 24-hour scheduling scheme is assumed for the analysis of the simulated system in order to clarify the performance of each power unit. Besides, the unity power factor is considered for all DGs, thus they just produce active power. The decision about power exchange between the MG and the utility, which is allowed at any hour in a day in order to more profitably exploit the market, is taken by $\mathrm{MG}$ central controller (MGCC). The data for the hourly active power of PV and WT, forecasted load demand and the utility power production bid, besides the entire bid data for all DGs along with the power market are available in [12]. PV and WT units do not consume any fuel at the times they produce electrical power during the day, consequently, the utility has to buy all electrical power produced by these units [12]. A Pareto-optimal set is attained for the two incompatible objectives (cost and emission) in each case. It is worth mentioning that applying the proposed approach for controlling the size of repository leads to the extraordinary fast convergence of the proposed MMOGWO that makes it superior among all other existing algorithms. The proposed method was implemented in MATLAB 8.1 and solved in a laptop with Core i5 CPU and 4GB RAM. The number of population and maximum iteration are both considered 100 .

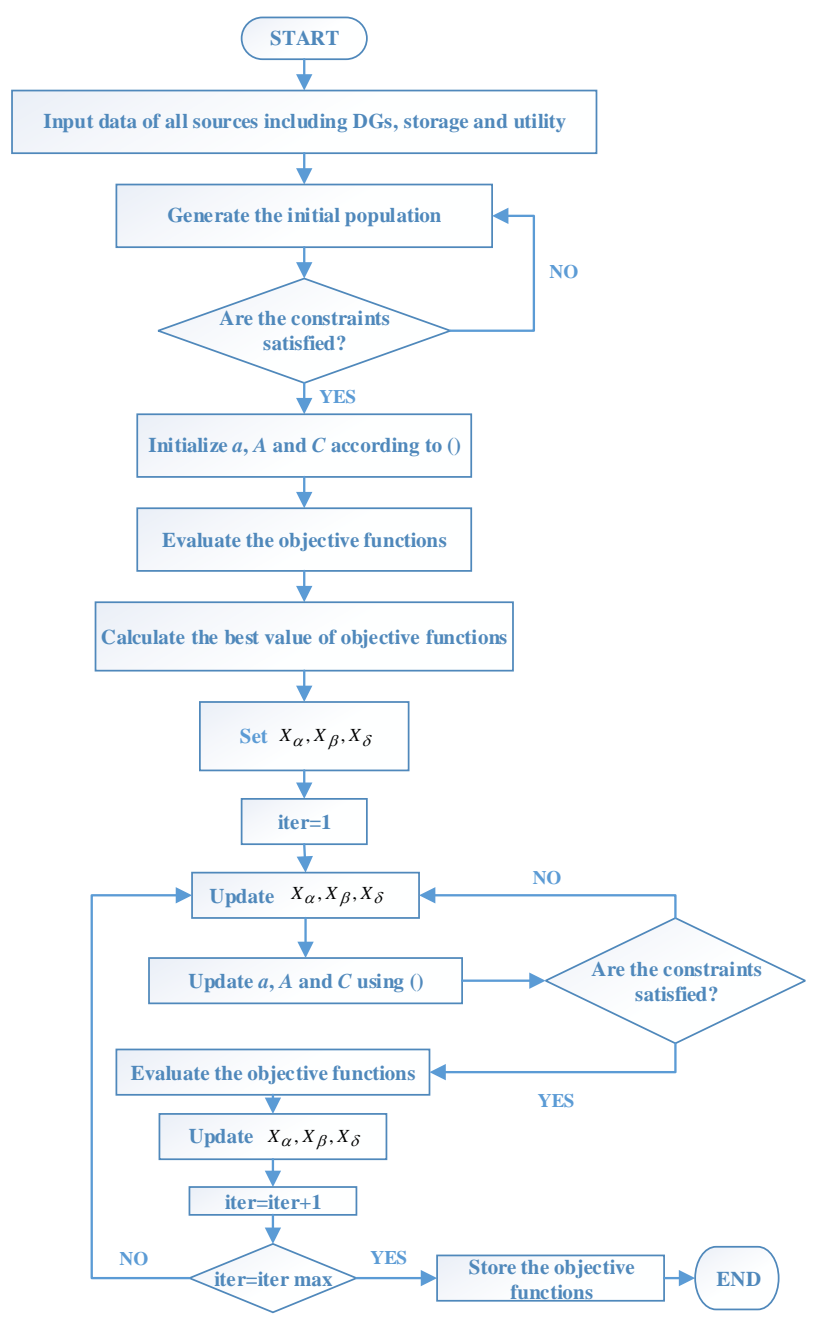

Fig. 1. Flowchart of the proposed algorithm.

\subsection{First case}

In this section, it is assumed that PV and WT (as non-dispatchable units) are in service at all hours during the day and are accepted to exploit at their maximum available output powers. This is while the ON\OFF states of dispatchable DGs (i.e. FC and MT) are considered. Therefore, in the algorithm process, the solutions for dispatchable units are compared with their minimum limit powers and if lower, the power will be put to zero. In this case no limits on the battery's initial charge is considered. The Pareto optimal front of this case is revealed in Figure 3, and results for three algorithms, including original 


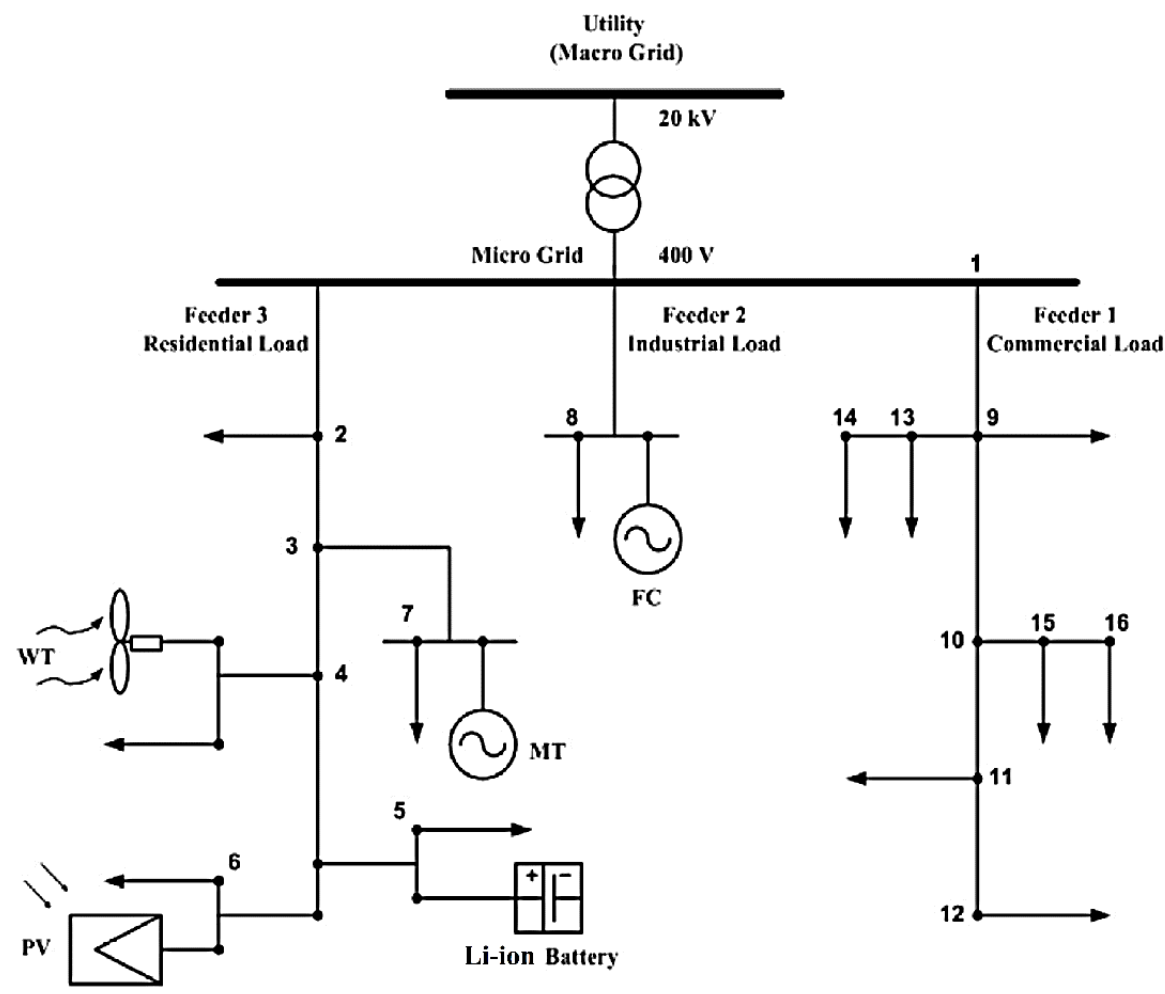

Fig 2. A typical MG test system [12].

GWO, PSO, and the proposed MMOGWO, are compared. The best compromised solution along with the values of the points where cost and emission are minimum are depicted. It is observed that in solving the MOOM problem, in addition to fast convergence, the proposed MMOGWO algorithm is able to properly find the points where the objective functions are in their minimum values, and the Pareto optimal front maintains between these points, while one deficiency of the two other algorithms in dealing with the considered MG energy management problem is the incapability to detect these points. Evidently, when the cost function is at its minimum value, $281.3 €$, the emission is $862.8 \mathrm{~kg}$. Besides, when emission decreases to $455.9 \mathrm{~kg}$, cost equals $857.2 €$. The best compromised solution is calculated according to the procedure described in section 3.3 where the cost and emission objective functions are $373.4 €$ and $566 \mathrm{~kg}$, respectively.

In order to justify the robustness and effectiveness of the proposed MMOGWO algorithm, the program is executed four times and results of these four different executions in the first case are revealed in Figure 4. Obviously, the achieved Pareto optimal sets in all four runs are approximately similar. It can be concluded that since the numbers of non-dominated solutions saved in the repository in different runs of the program, which are $150,150,147$ and 149 respectively for the first, second, third and fourth runs, are very close, the proposed algorithm is robust. Thereupon, not only can the proposed algorithm increase the convergence speed but also it decreases quiescence which results in getting away from local optimums. Consequently, the proposed MMOGWO proposes more robust and qualified solutions.

The power dispatch in the best compromised solution obtained using MMOGWO in this case is presented in Tables 3 in details. From these results, it is concluded that all equality and inequality constraints are satisfied. In the dispatch of the battery, when the battery is charging the values of power are negative, while during the discharging hours the values of power are positive. However, for the utility the negative values are representative of delivering energy to the upstream network, while the positive values are related to the times when energy is purchased from the upstream network. According to Table 3, since there is no limit on the battery charge, and the bid of the battery is less than other units, it sells power up to its maximum value in all hours of the day. Additionally, since the price of FC is less than MT, the purchased power from FC in different hours of the day is 
more. The MT power is limited on its permissible minimum value in most of hours because of the high price of power in comparison with other units.

As is expected, it is evidently obvious from Table 4 that when cost is the only considered objective function, it is significantly lower (which is equal to $269.85 €$ ) in comparison with the case that the ON/OFF states of dispatchable units is not considered where it is $278.25 €$. According to Table 4 , in the first 8 hours of the day, it is more economical when MT is in OFF mode.

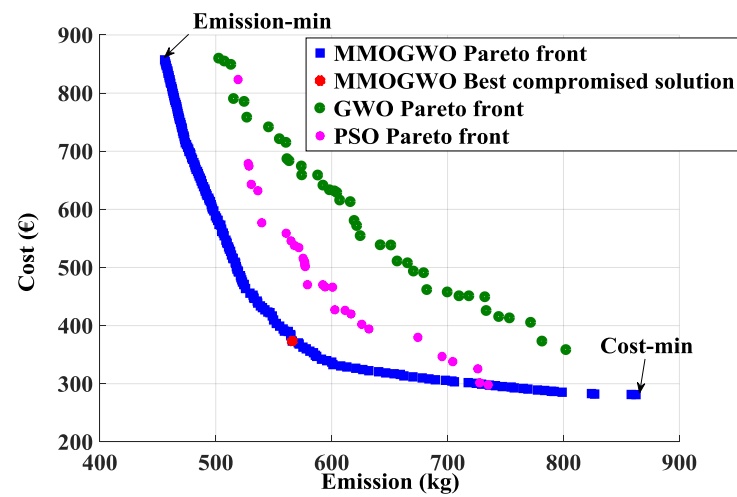

Fig. 3. Comparison of the objective functions Pareto optimal fronts of MMOGWO, original GWO and PSO algorithms for the first case.

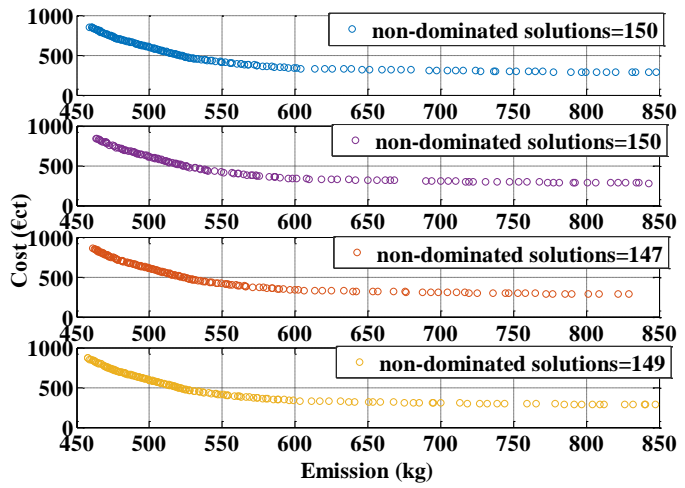

Fig. 4. Four different executions of MMOGWO algorithm (first case).

\subsection{Second case}

In the second scenario the battery initial charge is expected to be zero. The Pareto optimal solutions for this case are illustrated in Figure 5, where the best compromised solution along with the points where the two objective functions are minimum for the proposed MMOGWO are revealed, besides a com- parison between three algorithms, original GWO, $\mathrm{PSO}$, and MMOGWO, is performed. The minimum cost is equal to $484 €$, while emission is $1114 \mathrm{~kg}$; in Emission-minimum, the emission is reduced to 950.9 $\mathrm{kg}$, while cost equals $944.4 €$. The conflict of the two objectives can be concluded from these obtained values. Consequently, the detailed dispatch of the best compromised solution for the second case is tabulated in Table 3. In this case, the best compromised solution is when the cost and emission objective functions are $643.7 €$ and $1026 \mathrm{~kg}$, respectively. It is obvious from Table 3 that in the first 7 hours of the day, the local load is supplied by purchasing from the utility grid and even from MT, while the energy surplus is stored in the battery. At hour 8 , the battery starts to discharge for the load being supplied, where in this case, the energy surplus is sold to the upstream network. At hour 13 energy is purchased from the upstream network since the market price is low and as the market price is at maximum value at hour 14 this stored energy is sold to the utility at the next hour. Then, at hour 16 the battery is completely discharged. From hour 17 to 24 during which the market price is approximately low, the power is purchased from the upstream network and the battery is not charged nor discharged. Since the price of FC is less than other units, it sells power up to its maximum value in all hours of the day.

$$
\text { Table } 3
$$

MMOGWO best compromised solution of the first and second cases.

\begin{tabular}{ccccc|cccc}
\hline \hline Power & \multicolumn{9}{c|}{ First case } & \multicolumn{4}{c}{ Second case } \\
\cline { 2 - 3 }$(\mathrm{kW})$ & MT & FC & Battery & Utility & MT & FC & Battery & Utility \\
hour & & & & & & & & \\
1 & 6.027 & 13.825 & 30 & 0.364 & 30 & 30 & -10.2 & 0.5 \\
2 & 6 & 12.232 & 30 & -0.017 & 29.8 & 30 & -12.3 & 0.7 \\
3 & 6.184 & 12.8 & 30 & -0.769 & 29.8 & 30 & -16.1 & 4.6 \\
4 & 6 & 13.22 & 30 & -0.005 & 29.8 & 30 & -21.6 & 11 \\
5 & 6 & 18.205 & 29.997 & 0.013 & 29.8 & 29.7 & -20.8 & 15.6 \\
6 & 6.258 & 26.024 & 30 & -0.197 & 30 & 30 & -15.6 & 17.7 \\
7 & 9.398 & 29.184 & 30 & -0.366 & 29.8 & 29.9 & -0.4 & 8.9 \\
8 & 13.513 & 30 & 30 & -0.018 & 29.5 & 30 & 13.1 & 0.9 \\
9 & 10.708 & 29.995 & 29.931 & -0.169 & 30 & 30 & 10.4 & 0.1 \\
10 & 30 & 30 & 30 & -20.62 & 30 & 30 & 30 & -20.6 \\
11 & 28.776 & 30 & 29.999 & -30 & 30 & 30 & -0.5 & -0.7 \\
12 & 21.64 & 30 & 30 & -30 & 30 & 29.9 & 20.4 & -28.7 \\
13 & 6 & 29.951 & 29.954 & -21.72 & 29.8 & 30 & -15.5 & -0.1 \\
14 & 18.58 & 30 & 30 & -30 & 30 & 30 & 18.6 & -30 \\
15 & 29.974 & 30 & 30 & -23.63 & 29.9 & 30 & 6.3 & 0.1 \\
16 & 29.996 & 29.991 & 29.999 & -15.52 & 30 & 30 & 14.1 & 0.4 \\
17 & 22.678 & 29.999 & 29.987 & 0.0004 & 29.9 & 30 & 0 & 22.7 \\
18 & 26.628 & 30 & 30 & -0.413 & 30 & 30 & 0 & 26.2 \\
19 & 28.648 & 29.999 & 29.99 & 0.0601 & 30 & 30 & 0 & 28.7 \\
20 & 26.174 & 29.882 & 29.928 & -0.768 & 30 & 30 & 0 & 25.2 \\
21 & 16.699 & 30 & 30 & 0 & 30 & 30 & 0 & 16.7 \\
22 & 9.706 & 30 & 30 & -0.007 & 30 & 30 & 0 & 9.7 \\
23 & 6 & 28.094 & 29.999 & -0.008 & 29.9 & 30 & 0 & 4.2 \\
24 & 6.046 & 19.381 & 29.974 & -0.016 & 25.6 & 29.8 & 0 & -0.1 \\
\hline
\end{tabular}




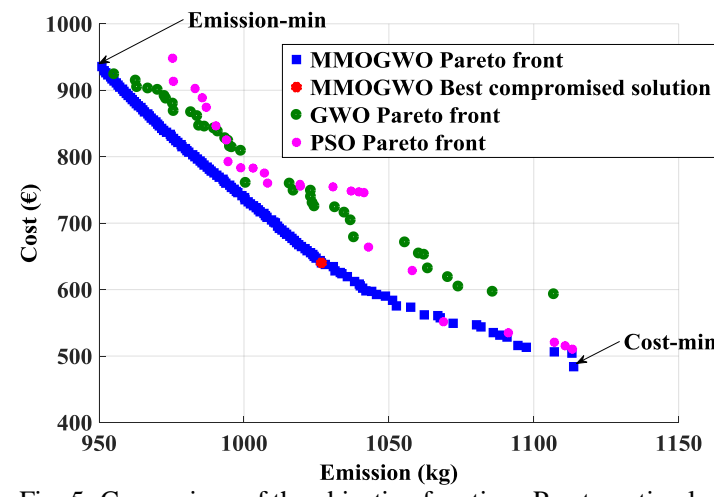

Fig. 5. Comparison of the objective functions Pareto optimal fronts of MMOGWO, original GWO and PSO algorithms for the second case.

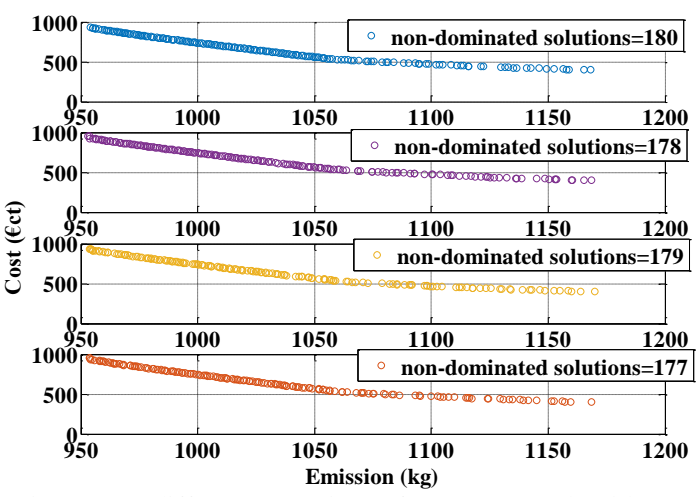

Fig. 6. Four different executions of MMOGWO algorithm (second case)

Table 4

MMOGWO optimal solution achieved in the first case where the cost is reduced to $269.85 €$

\begin{tabular}{|c|c|c|c|c|}
\hline $\begin{array}{l}\text { Power } \\
\text { (kWh) }\end{array}$ & MT & FC & Battery & Utility \\
\hline \multicolumn{5}{|l|}{ hour } \\
\hline 1 & 0 & 20.171 & 0.0445 & 30 \\
\hline 2 & 0 & 17.924 & 0.2911 & 30 \\
\hline 3 & 0 & 18.145 & 0.0699 & 30 \\
\hline 4 & 0 & 19.129 & 0.1158 & 29.9706 \\
\hline 5 & 0 & 24.159 & 0.0881 & 29.9679 \\
\hline 6 & 0 & 29.906 & 2.1815 & 29.9976 \\
\hline 7 & 0 & 29.992 & 8.3458 & 29.8773 \\
\hline 8 & 0 & 30 & 18.6006 & 24.8944 \\
\hline 9 & 29.991 & 29.999 & 30 & -19.5251 \\
\hline 10 & 30 & 30 & 30 & -20.615 \\
\hline 11 & 28.817 & 30 & 29.9576 & -30 \\
\hline 12 & 21.787 & 29.856 & 29.9964 & -29.9997 \\
\hline 13 & 14.186 & 29.999 & 30 & -29.9992 \\
\hline 14 & 18.729 & 29.855 & 29.9959 & -29.9997 \\
\hline 15 & 29.993 & 30 & 30 & -23.6533 \\
\hline 16 & 30 & 30 & 29.9995 & -15.5295 \\
\hline 17 & 30 & 30 & 30 & -7.335 \\
\hline 18 & 0 & 30 & 30 & 26.215 \\
\hline 19 & 0 & 30 & 28.7922 & 29.9058 \\
\hline 20 & 26.429 & 30 & 30 & -1.2142 \\
\hline 21 & 30 & 30 & 30 & -13.3005 \\
\hline 22 & 29.905 & 29.942 & 29.9868 & -20.1343 \\
\hline 23 & 0 & 30 & 4.085 & 30 \\
\hline 24 & 0 & 25.188 & 0.2147 & 29.982 \\
\hline
\end{tabular}

In Figure 6 a comparison between four executions of the algorithm in the second case is shown. Obviously, the achieved Pareto optimal sets in all four runs are approximately similar. As is obvious from Figure 6, the numbers of non-dominated solutions saved in the repository in different runs of the program, which are 180, 178, 179 and 177 respectively for the first, second, third and fourth runs, are very close. Consequently, the robustness of the proposed MMOGWO algorithm in the second case can be justified through the results of Figure 6.

When the initial charge of the battery is zero, a strict restriction on the battery charge and discharge is put on. Consequently, the most adjustable scenario is when the battery has initial charge. Because of the more realistic performance of the battery in the second case, more power is purchased from MT, comparatively. In both cases, in most hours a large amount of power is bought from FC since it is less expensive. In first hours of the day where the market price is lower, the system operator purchases power from the utility. This power can be utilized in order to supply local loads or can be stored in the storage device. However, in peak hours, the stored power can be sold to the utility in a much higher price.

The superiority of an optimization algorithm in solving multi-objective problems is concluded from the appropriate and fast convergence, while attaining exact Pareto front. Moreover, the robustness of an optimization algorithm can be another criterion in order to prove the effectiveness of the method. Consequently, as is concluded from the obtained results and by comparing the Pareto optimal sets of three algorithms in solving the MOOM problem, the priority of the proposed MMOGWO algorithm along with its robustness and accuracy is justified. Accordingly, it is proved that the proposed MMOGWO has successfully fulfilled these assumed criteria, and it can significantly achieve the exceptional solution in comparison with other methods in solving MOOM problem of an MG.0

\section{Conclusions}

The new MMOGWO algorithm was proposed in order to deal with the multi-objective optimal operation management in a typical MG. Three modifications were added to the original GWO which result in more accurate and faster performance of the suggested approach. The variable population size resulted from the first modification avoids trapping in local 
optima, while the two other modifications were augmented to the mutation procedure in order to increase the convergence speed and robustness of the algorithm. Additionally, a novel method was applied for controlling the size of repository which generates a well-distributed Pareto front in a very low computational time. As a result, the accuracy and speed of the algorithm will improve. It is obvious from the results that an exceptional Pareto optimal set was achieved while applying the presented method comparing with original GWO and PSO algorithms. Two different scenarios were considered in order to justify the effectiveness of MMOGWO. Simulation results manifest that the proposed method is able to deal with mixed-integer problems. Future works can include the following:

1. Investigating the stochastic MG optimal operation management while considering the uncertainties of renewable resources using the proposed algorithm.

2. Studying the effects of elements of the future smart grid, such as electric vehicles, in the considered MG energy management.

\section{Acknowledgement}

This work was supported in part by Royal Academy of Engineering Distinguished Visiting Fellowship under Grant DVF1617\6\45.

\section{References}

[1] M. Vosoogh, M. Kamyar, and A. Akbari, "A novel modification approach based on MTLBO algorithm for optimal management of renewable micro-grids in power systems." Journal of Intelligent \& Fuzzy Systems vol. 27, (1), pp. 465-473, 2014.

[2] H. R. Baghaee, M. Mirsalim, and G. B. Gharehpetian, "Multi-objective optimal power management and sizing of a reliable wind/PV microgrid with hydrogen energy storage using MOPSO," Journal of Intelligent \& Fuzzy Systems vol. 32, (3), pp. 1753-1773, 2017.

[3] W. Gu, Z. Wu, R. Bo, W. Liu, G. Zhou, W. Chen, et al., "Modeling, planning and optimal energy management of combined cooling, heating and power microgrid: A review," International Journal of Electrical Power \& Energy Systems, vol. 54, pp. 26-37, 2014.

[4] N. Javidtash, M. Jabbari, T. Niknam, and M. Nafar, "A novel mixture of non-dominated sorting genetic algorithm and fuzzy method to multi-objective placement of distributed generations in Microgrids." Journal of Intelligent \& Fuzzy Systems Preprint pp. 1-8, 2017.

[5] H. R. Kamankesh, and G. A. Vassilios, "A sufficient stochastic framework for optimal operation of micro-grids considering high penetration of renewable energy sources and electric vehicles." Journal of Intelligent \& Fuzzy Systems vol. 32, (1), pp. 373-387, 2017

[6] A. R. Abbasi, S. Abbasi, J. Ansari, and E. Rahmani, "Effect of plug-in electric vehicles demand on the renewable microgrids," Journal of Intelligent \& Fuzzy Systems vol. 29, (5), pp. 1957-1966, 2015.

[7] T. A. Nguyen, and M. L. Crow, "Stochastic optimization of renewable-based microgrid operation incorporating battery operating cost," IEEE Transactions on Power Systems, vol. 31, pp. 2289-2296, 2016

[8] K. W. Hu, and C. M. Liaw, "Incorporated operation control of DC microgrid and electric vehicle," IEEE Transactions on Industrial Electronics, vol. 63, pp. 202-215, 2016.

[9] M. Marzband, E. Yousefnejad, A. Sumper, and J. L. Domínguez-García, "Real time experimental implementation of optimum energy management system in standalone microgrid by using multi-layer ant colony optimization," International Journal of Electrical Power \& Energy Systems, vol. 75, pp. 265-274, 2016.

[10] R. Jabbari-Sabet, S.-M. Moghaddas-Tafreshi, and S.-S. Mirhoseini, "Microgrid operation and management using probabilistic reconfiguration and unit commitment," International Journal of Electrical Power \& Energy Systems, vol. 75, pp. 328-336, 2016.

[11] M. H. Moradi, M. Abedini, and S. M. Hosseinian, "Optimal operation of autonomous microgrid using HS-GA," International Journal of Electrical Power \& Energy Systems, vol. 77 pp. 210-220, 2016

[12] S. Parhoudeh, A. Baziar, A. Mazareie, and A. Kavousi-Fard, "A novel stochastic framework based on fuzzy cloud theory for modeling uncertainty in the micro-grids," International Journal of Electrical Power \& Energy Systems, vol. 80, pp. 73-80, 2016

[13] R. Cheng, T. Rodemann, M. Fischer, M. Olhofer, and Y. Jin, "Evolutionary Many-objective Optimization of Hybrid Electric Vehicle Control: From General Optimization to Preference Articulation, " IEEE Transactions on Emerging Topics in Computational Intelligence, vol. 1, (2), pp. 97-111, 2017.

[14] M. Zaman, S. M. Elsayed, T. Ray, and R. A. Sarker, "Evolutionary algorithms for dynamic economic dispatch problems," IEEE Transactions on Power Systems, vol. 31, pp. 1486-1495, 2016.

[15] F. A. Mohamed and H. N. Koivo, "Multiobjective optimization using Mesh Adaptive Direct Search for power dispatch problem of microgrid," International Journal of Electrical Power \& Energy Systems, vol. 42, pp. 728-735, 2012.

[16] T. Lv, Q. Ai, and Y. Zhao, "A bi-level multi-objective optimal operation of grid-connected microgrids," Electric Power Systems Research, vol. 131, pp. 60-70, 2016.

[17] A. Baziar and A. Kavousi-Fard, "Considering uncertainty in the optimal energy management of renewable micro-grids including storage devices," Renewable Energy, vol. 59, pp. 158-166, 2013.

[18] A. Kavousi-Fard, A. Abunasri, A. Zare, and R. Hoseinzadeh, "Impact of plug-in hybrid electric vehicles charging demand on the optimal energy management of renewable microgrids," Energy, vol. 78, pp. 904-915, 2014.

[19] G. Aghajani, H. Shayanfar, and H. Shayeghi, "Presenting a multi-objective generation scheduling model for pricing demand response rate in micro-grid energy management," Energy Conversion and Management, vol. 106, pp. 308-321, 2015.

[20] S. Mirjalili, S. M. Mirjalili, and A. Lewis, "Grey wolf optimizer," Advances in Engineering Software, vol. 69, pp. 46-61, 2014. 
[21] S. Rajasomashekar and P. Aravindhababu, "Biogeography based optimization technique for best compromise solution of economic emission dispatch," Swarm and Evolutionary Computation, vol. 7, pp. 47-57, 2012.

[22] S. Sharma, S. Bhattacharjee, and A. Bhattacharya, "Grey wolf optimisation for optimal sizing of battery energy storage device to minimise operation cost of microgrid," IET Generation, Transmission \& Distribution, vol. 10, pp. 625-637, 2016. 